Pak. j. sci. ind. res. Ser. A: phys. sci. 2014 57(2) 95-102

\title{
Noise Pollution - A Case Study of Rawalpindi City, Pakistan
}

\author{
Younas Kalim*, Tahseen Aslam and Hajra Masood \\ Natinal Physical and Standards Laboratory, PCSIR, 16-H/9, Islamabad, Pakistan \\ (received February 1, 2013; revised May 2, 2013; accepted June 26, 2013)
}

\begin{abstract}
In this study, noise level was measured during day time in 88 different locations of the Rawalpindi city, Pakistan, which included roads, choaks, residential areas, educational institutions, hospitals, railway stations,airport, bus stands, shopping plazas and markets. The noise measurements were performed with a calibrated sound level meter. Study finds that overall minimum and maximum noise levels for the main roads and choaks were 55.4 and $101.9 \mathrm{~dB}(\mathrm{~A})$, for residential areas 38.80 and $91.0 \mathrm{~dB}(\mathrm{~A})$, for educational institutions 60.0 and $94.4 \mathrm{~dB}(\mathrm{~A})$, for hospital 45.1 and $84.4 \mathrm{~dB}(\mathrm{~A})$, for railway stations, airport, bus stands 59.2 and $102.5 \mathrm{~dB}(\mathrm{~A})$ and for shopping plazas, markets 53.8 and $81.2 \mathrm{~dB}(\mathrm{~A})$, respectively. The result of the study revealed that the noise level surpassed the prescribed NEQS limits as well as WHO guideline values for noise in specific environments in all areas under study, which can cause harmful effects on human health, animals and the environment.
\end{abstract}

Keywords: noise pollution, sound level, environment

\section{Introduction}

Noise pollution can be defined as intrusive noise that disrupts, distracts, or detracts from regular functioning. Noise pollution is not new, but it has become more problematic with the developments associated with industrialisation and urbanisation. Between 1987 and 1997, community noise levels in the United States were estimated to have increased by $11 \%$ and were predicted to continue increasing at that rate or more (Staples, 1997). The response of the human ear to sound depends both on the sound frequency (measured in Hertz, Hz) and the sound pressure, measured in decibels $(\mathrm{dB})$. A normal ear in healthy young person can detect sounds with frequencies from $20 \mathrm{~Hz}$ to $20,000 \mathrm{~Hz}$. Noise measurements are expressed by the term sound pressure level (SPL), which is logarithmic ratio of the sound pressure to a reference pressure and is expressed as a dimensionless unit of power, the decibel $(\mathrm{dB})$. The reference level is 0.0002 microbars, the threshold of human hearing.

$$
\text { Decibel } \mathrm{L}_{\text {eq }}=10 \log 10 \mathrm{~L} / \mathrm{L}^{\circ}
$$

where:

$\mathrm{L}_{\mathrm{eq}}=$ equivalent noise level; $\mathrm{L}=$ sound intensity; $\mathrm{L}^{\circ}=$ reference level

Sound level becomes noise when it crosses the $70 \mathrm{~dB}$ mark. Noise levels above 80 decibels produce damaging effects to the ear. It can cause irreparable damage

*Author for correspondence; E-mail: ykalim@yahoo.com and lead to permanent hearing loss when noise level is above 100 decibels for a considerable period of time.

For measurement of noise emission a sound level meter is used. A measure of the level of sound is called the decibel. The zero of the decibel scale is the hearing threshold. Sounds at 0-10 decibel are so quiet that they are almost impossible to hear, while at the top end of the scale, at around 150 decibel, it can damage eardrums. (http://www.epa.vic.gov.au).

There are many sources of noise pollution such as different machines used in industries, horns and whistles of railway engines, switching and shunting operation in rail yards, airoplanes etc., that have significant negative impact on human health. Noise pollution is a significant environmental problem in many rapidly urbanising areas. This problem is not properly recognised despite the fact that it is steadily growing in developing countries (Murthy et al., 2007). Traffic noise is the biggest source of noise pollution, especially in urban areas. For example an average noise level produced by vehicular traffic on roads of Karachi city, Pakistan, is $90 \mathrm{~dB}$ (A) (Khan et al., 2010). Noise level of $112.3 \mathrm{~dB}(\mathrm{~A})$ was observed in a study conducted in Tangail Municipal area, Bangladesh. Noise levels in this study area exceeded the recommended level by WHO at 34 out of 47 measuring points (Mia et al., 2012). Noise-induced hearing loss (NIHL) in humans is a major problem stemming from noise pollution as well as heart-related, 
respiratory, neurological and other physiological problems. Stress, high blood pressure, anger and frustration, lower resistance to disease and infection, circulatory problems, ulcers, asthma, colitis, headaches, gastrointestinal disorders, and many other physiological and psychological problems have been linked directly to noise. In addition, children have been shown to suffer from slower language development and disruption of learning as a result of noise.

A study carried out at ENT Department, Sir Ganga Ram Hospital, Lahore, found that public transport drivers are exposed to excess noise on roads in Lahore and $65 \%$ of them had noise induced hearing loss (NIHL). 25\% had normal hearing threshold and 10\% had disabling hearing loss (Aslam et al., 2008). The hearing ability of the inhabitants of Dhaka city, Bangladesh, has reduced and they are suffering from permanent deafness due to noise pollution (Alam et al., 2001). In developed countries, as many as four to five million people, i.e., $12-15 \%$ of all employed people, are exposed to noise levels of 85 decibels or more (WHO, 2001). More than five million children in the United States, ages six to nineteen, suffer from noise-induced hearing impairment (Havas, 2006). In the exposure to noise impaired children's reading comprehension and caused a delay in reading skills development (Clark and Stansfeld, 2005). In children in noisier neighborhoods were shown to suffer from increased stress and diminished motivation (Evans et al., 2001).

In the present study, noise level was estimated in Rawalpindi city, Pakistan. Rawalpindi is situated at latitude $33.60^{\circ} \mathrm{N}$ and longitude $73.04{ }^{\circ} \mathrm{E}$, in the province of Punjab. It is the fourth largest city in Pakistan after Karachi, Lahore and Faisalabad. Rawalpindi city is divided into two tehsils:

Potohar (southern Rawalpindi)

Rawal (northern Rawalpindi)

The population of Rawalpindi city is approximately 19, 91,656 (World Gazetteer, 2010). The city is home to several industries and factories. The total area of the city is approximately 108.08 square $\mathrm{km}$ (Wiki/World Gazetteer). Murree road and Peshawar road are main roads of the city. Murree road has been a hot spot for various political and social events. Kashmir road, Haider road, Bank road, Hospital road, Jinnah road, Said pur road, IJP road, Rawal road, Tipu road, Tench road, Misrial road, Adiala road and Airport road are other important roads of the city. Main bazaars and markets of the city are Raja bazaar,Tench bazaar, Moti bazaar, Kashmiri bazaar, Sarrafa bazaar, Saddar area, Commercial market, Westridge market, Chah Sultan market and Kamran market. Number of shopping plazas and centers have been constructed on both sides of Murree road.

Being twin city of Islamabad, the capital of Pakistan, there is a rapid expansion in Rawalpindi city area. This expansion is mostly unplanned. Most of the areas particularly to the urban side are subjected to unacceptable noise conditions due to construction, manufacturing, traffic and recreational activities. A comprehensive national survey has not been conducted to assess the level of noise pollution in big cities of Pakistan. Present study was conducted to assess the noise level in the Rawalpindi city as well as its main causes. It will certainly help implementing agencies to understand the severity of the noise pollution problem of the city and take proper remedial measures.

\section{Materials and Methods}

In this study, sound level meter type 2240, Bruel \& Kjaer, Denmark was used to measure the noise level. The microphone converts sound to an equivalent electric signal, which is processed by the instrument. Processing includes applying frequency and time weightings to the signal as specified by international standards, IEC 61672-1 to which this metre conforms.

Frequency weighting adjusts how the sound level meter responds to different sound frequencies. This is necessary because the human ear's sensitivity to sound varies according to the sound's frequency. The most common frequency weighting in use is Aweighting, which adjusts a signal in a way that best resembles the human ear's response at medium-range levels. It is the weighting required for nearly all environmental and workplace noise measurements and is specified in international and national standards and guidelines. All of Type 2240's measurement parameters apply A-weighting, except for measurement of peak levels, where the $\mathrm{C}$ frequency weighting is applied.

Time weighting specifies, how the sound level metre reacts to changes in sound pressure. It is an exponential 
averaging of the fluctuating signal, providing an easyto-read value. Type 2240 applies the Fast, or F time weighting, which is required weighting according to the vast majority of international and national standards and guidelines. Once the signal is processed through the weighting filters, the resulting sound pressure level is displayed in decibels $(\mathrm{dB})$ on the instruments display.

All the measurements were taken at different times during day time (between 10 am to $3 \mathrm{pm}$ ) from August to October, 2012. Noise level was noted ten times at each point then averaged through summation of all values divided by the number of observation. At the time of collecting data of noise, values of frequent peaks were measured. The minimum and maximum levels of noise were recorded in each observation on the basis of maximum and minimum peaks noted during data recording. Standard deviation was calculated from ten observations obtained from each point.

\section{Results and Discussion}

In this study noise level was measured during day time in 85 points in different areas of the Rawalpindi city, Pakistan. The minimum, maximum and average noise levels were measured in $\mathrm{dB}(\mathrm{A})$ and standard deviation of all points is shown in Tables 1-6. Minimum and maximum noise levels at monitoring areas are shown in Fig. 1.

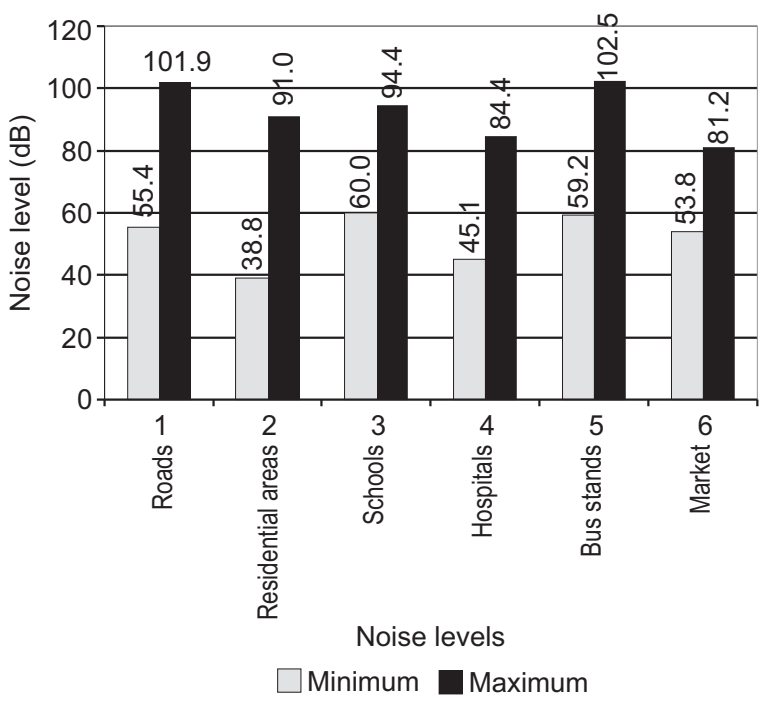

Fig. 1. Noise level (minimum and maximum).
The result of the study revealed that the noise level surpassed the prescribed NEQS (Table 7) as well as WHO guideline values (Table 8) in all areas under study, which can cause harmful effects on human health, animals and the environment. The study finds that overall minimum and maximum noise levels for main roads and choaks is 55.4 and $101.9 \mathrm{~dB}(\mathrm{~A})$, with mean values $61.6 \pm 4.8-83.7 \pm 5.88 \mathrm{~dB}(\mathrm{~A})$, higher than NEQS and WHO guideline value of 75(NEQS Table 7) and $70 \mathrm{~dB}$ (WHO guidelines, Table 8), respectively. Minimum and maximum noise level for residential areas is 38.8 and $91.0 \mathrm{~dB}(\mathrm{~A})$, with mean values $47.7 \pm 5.2-78.2 \pm 4.9 \mathrm{~dB}(\mathrm{~A})$. It is also on higher side as compared to NEQS and WHO limits of 55 dB (NEQS Table 7, WHO guidelines Table 8) set for outdoor living areas. At educational institutions minimum and maximum noise level is 60.0 and $94.4 \mathrm{~dB}(\mathrm{~A})$, with mean values $65.7 \pm 3.7-85.0 \pm 4.3$ $\mathrm{dB}(\mathrm{A})$, respectively. It is about 11-35 $\mathrm{dB}$ high than NEQS and WHO guideline value $50 \mathrm{~dB}$ (NEQS Table 7 and WHO guidelines, Table 8) set for school playground outdoor and silence zone. For hospitals minimum and maximum noise levels observed are

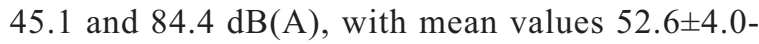
$76.3 \pm 4.6 \mathrm{~dB}(\mathrm{~A})$, which is on higher side as compared to WHO guideline values of $30 \mathrm{~dB}$ (WHO guidelines, Table 8) set for hospital, ward rooms, indoor as well as NEQS for silence zone, which is $50 \mathrm{~dB}$ (NEQS Table 7). It is an alarming sign for hospitals management. For railway stations, airport, and bus stands observed minimum and maximum noise level is 59.2 and $102.5 \mathrm{~dB}(\mathrm{~A})$, with mean values $56.4 \pm 9.3$ $80.2 \pm 2.4 \mathrm{~dB}(\mathrm{~A})$, higher than WHO as well as NEQS limits for traffic. For shopping plazas, markets minimum and maximum noise level observed is 53.8 and $81.2 \mathrm{~dB}(\mathrm{~A})$, with mean values $58.8 \pm 5.2-$ $78.7 \pm 1.5 \mathrm{~dB}(\mathrm{~A})$, respectively. It is also higher than WHO guideline value of $70 \mathrm{~dB}$ (WHO guidelines, Table 8) set for industrial, commercial, shopping areas as well as NEQS limit of $65 \mathrm{~dB}$ (NEQS Table 7) set for commercial areas.

\section{Conclusion}

The results of the study revealed that the noise level surpassed the prescribed NEQS limits as well as WHO guideline values of noise level for specific environments, in all areas under study in Rawlpindi city which 
Table 1. Results from noise measurements on roads and choaks

\begin{tabular}{|c|c|c|c|c|c|c|c|c|c|c|c|c|c|c|}
\hline Roads and choaks & 1 & 2 & 3 & 4 & 5 & 6 & 7 & 8 & 9 & 10 & Max & Min & Mean & SD \\
\hline IJP Peshawar road & 69.0 & 76.8 & 75.8 & 70.3 & 71.6 & 68.2 & 88.2 & 74.8 & 75.1 & 80.1 & 88.2 & 68.2 & 75.0 & 6.0 \\
\hline IJP Toll plaza & 80.5 & 92.3 & 73.7 & 82.1 & 94.2 & 81.9 & 75.8 & 70.0 & 82.5 & 77.9 & 94.2 & 70.0 & 81.1 & 7.6 \\
\hline IJP Carriage factory & 81.3 & 80.9 & 72.9 & 86.5 & 89.7 & 78.0 & 82.2 & 83.2 & 80.8 & 70.2 & 89.7 & 70.2 & 80.6 & 5.8 \\
\hline IJP Pir wadhai more & 80.0 & 89.4 & 76.0 & 84.1 & 101 & 71.2 & 77.1 & 76.0 & 80.0 & 89.0 & 101.0 & 71.2 & 82.4 & 8.8 \\
\hline IJP Pindora choak & 84.8 & 75.5 & 78.1 & 89.7 & 98.1 & 82.0 & 69.9 & 74.4 & 81.1 & 90.1 & 98.1 & 69.9 & 82.4 & 8.5 \\
\hline Agha shahi avenue-9 & 72.4 & 70.6 & 75.1 & 72.0 & 76.6 & 78.2 & 69.2 & 66.6 & 78.1 & 71.0 & 78.2 & 66.6 & 73.0 & 3.9 \\
\hline Faizabad choak & 70.1 & 69.1 & 66.2 & 69.0 & 71.3 & 68.2 & 82.6 & 70.9 & 75.2 & 68.5 & 82.6 & 66.2 & 71.1 & 4.7 \\
\hline 6th road choak & 67.7 & 69.0 & 68.1 & 69.1 & 66.0 & 65.1 & 86.9 & 68.9 & 75.6 & 71.6 & 86.9 & 65.1 & 70.8 & 6.4 \\
\hline $\begin{array}{l}\text { Muslim town } \\
\text { sadiqabad choak }\end{array}$ & 70.2 & 66.8 & 68.2 & 75.2 & 65.9 & 78.8 & 80.1 & 70.0 & 65.7 & 80.0 & 80.1 & 65.7 & 72.1 & 5.9 \\
\hline Chandni choak flyover & 77.7 & 66.4 & 64.0 & 63.3 & 62.8 & 69.4 & 61.9 & 68.9 & 101.9 & 98.2 & 101.9 & 61.9 & 73.5 & 14.8 \\
\hline $\begin{array}{l}\text { Sirvice road } \\
\text { dhoke kashmirian }\end{array}$ & 69.3 & 67.8 & 72.2 & 67.4 & 68.4 & 69.9 & 67.0 & 71.9 & 61.9 & 66.8 & 72.2 & 61.9 & 68.3 & 2.9 \\
\hline Banni choak & 72.3 & 71.9 & 68.6 & 68.9 & 69.8 & 76.1 & 73.8 & 74.0 & 72.6 & 70.2 & 76.1 & 68.6 & 71.8 & 2.4 \\
\hline Asghar maal choak & 80.0 & 77.7 & 75.5 & 80.6 & 76.7 & 78.2 & 73.1 & 79.7 & 74.7 & 77.2 & 80.6 & 73.1 & 77.3 & 2.4 \\
\hline Pindora chungi choak & 72.5 & 71.4 & 75.4 & 78.4 & 68.2 & 70.6 & 73.1 & 60.7 & 58.9 & 61.3 & 78.4 & 58.9 & 69.1 & 6.7 \\
\hline Siddiqui choak & 77.1 & 74.0 & 68.1 & 69.5 & 69.0 & 71.0 & 72.7 & 70.6 & 69.7 & 68.3 & 77.1 & 68.1 & 71.0 & 2.9 \\
\hline Committee choak & 77.5 & 76.5 & 80.0 & 92.5 & 79.5 & 81.1 & 78.3 & 84.1 & 78.8 & 76.1 & 92.5 & 76.1 & 80.4 & 4.8 \\
\hline $\begin{array}{l}\text { PAF Auditorium choak } \\
\text { chaklala base }\end{array}$ & 72.1 & 72 & 66.8 & 65.4 & 72.8 & 76.8 & 69.1 & 63.2 & 59.8 & 78.2 & 78.2 & 59.8 & 69.6 & 5.9 \\
\hline $\begin{array}{l}\text { RDA/WASA } \\
\text { Liaqat bagh murree road }\end{array}$ & 67.2 & 68.8 & 69.5 & 66.5 & 90.5 & 71.5 & 76.3 & 78.9 & 81.5 & 85.5 & 90.5 & 66.5 & 75.6 & 8.3 \\
\hline Moti mahal murree road & 73.7 & 75.6 & 75.0 & 94.8 & 87.6 & 90.1 & 82.6 & 80.5 & 83.5 & 89.4 & 94.8 & 73.7 & 83.3 & 7.2 \\
\hline Mareer hasan choak & 68.9 & 85.5 & 72.5 & 83.5 & 77.4 & 70.8 & 69.5 & 68.0 & 70.0 & 92.2 & 92.2 & 68.0 & 75.8 & 8.5 \\
\hline Kachahri choak & 72.9 & 68.9 & 70.5 & 71.9 & 68.6 & 71.5 & 90.2 & 89.0 & 80.0 & 73.5 & 90.2 & 68.6 & 75.7 & 8.0 \\
\hline Ammar choak & 68.3 & 71.5 & 72.2 & 69.8 & 75.5 & 81.6 & 61.8 & 60.7 & 79.9 & 62.4 & 81.6 & 60.7 & 70.4 & 7.3 \\
\hline $\begin{array}{l}\text { Ayub park } \\
\text { Main gate GT road }\end{array}$ & 66.4 & 69.1 & 70.2 & 65.4 & 67.3 & 64.8 & 68.8 & 69.8 & 74.1 & 77.5 & 77.5 & 64.8 & 69.3 & 3.9 \\
\hline Chungi no.22 choak & 86.4 & 81.6 & 80.9 & 75.6 & 81.4 & 77.5 & 86.9 & 82.6 & 79.4 & 79.0 & 86.9 & 75.6 & 81.1 & 3.6 \\
\hline $\begin{array}{l}\text { Tench bhata } \\
\text { dispensary road }\end{array}$ & 77.6 & 83.5 & 83.9 & 91.8 & 81.1 & 84.1 & 82.2 & 80.5 & 76.8 & 95.6 & 95.6 & 76.8 & 83.7 & 5.9 \\
\hline $\begin{array}{l}\text { Kalma choak } \\
\text { Dhoke syyedan }\end{array}$ & 75.0 & 69.7 & 64.4 & 70.4 & 68.7 & 70.3 & 74.4 & 80.1 & 67.3 & 72.2 & 80.1 & 64.4 & 71.3 & 4.4 \\
\hline $\begin{array}{l}\text { Fawara choak } \\
\text { raja bazaar }\end{array}$ & 82.9 & 77.4 & 75.4 & 71.7 & 84.6 & 79.3 & 80.0 & 87.9 & 90.1 & 70.9 & 90.1 & 70.9 & 80.0 & 6.5 \\
\hline Dinghi khui raja bazaar & 71.5 & 70.7 & 79.1 & 66.5 & 66.9 & 78.4 & 68.9 & 70.0 & 69.2 & 81.5 & 81.5 & 66.5 & 72.3 & 5.4 \\
\hline Bohar bazaar choak & 83.2 & 80.5 & 74.1 & 79.3 & 74.8 & 75.6 & 81.9 & 80.2 & 78.6 & 88.6 & 88.6 & 74.1 & 79.7 & 4.4 \\
\hline $\begin{array}{l}\text { F.G Boys school } \\
\text { Peshawar road }\end{array}$ & 85.2 & 84.4 & 73.6 & 69.1 & 74.4 & 70.4 & 76.0 & 69.7 & 78.4 & 78.8 & 85.2 & 69.1 & 76.0 & 5.7 \\
\hline $\begin{array}{l}\text { Chuhar choak } \\
\text { Peshawar road }\end{array}$ & 66.8 & 67.4 & 59.4 & 68.1 & 55.4 & 58.2 & 60.1 & 60.0 & 64.2 & 55.9 & 68.1 & 55.4 & 61.6 & 4.7 \\
\hline Dhoke ratta bazaar & 80.8 & 80.2 & 70.8 & 67.3 & 71.1 & 69.8 & 72.6 & 73.4 & 71.3 & 73.7 & 80.8 & 67.3 & 73.1 & 4.3 \\
\hline
\end{tabular}


Table 2. Results from noise measurements on residential areas

\begin{tabular}{|c|c|c|c|c|c|c|c|c|c|c|c|c|c|c|}
\hline Area & 1 & 2 & 3 & 4 & 5 & 6 & 7 & 8 & 9 & 10 & Max & Min & Mean & $\mathrm{SD}$ \\
\hline A-Block satellite town & 77.9 & 64.4 & 67.5 & 66.4 & 72.2 & 68.0 & 65.7 & 71.5 & 67.4 & 74.3 & 77.9 & 64.4 & 69.5 & 4.3 \\
\hline B-Block satellite town & 58.6 & 59 & 62.1 & 61.4 & 54.6 & 55.8 & 62.5 & 50.8 & 56.3 & 64.2 & 64.2 & 50.8 & 58.5 & 4.2 \\
\hline D-Block satellite town & 78.1 & 74.4 & 73.6 & 75.3 & 76.6 & 69.0 & 70.0 & 68.2 & 69.1 & 67.3 & 78.1 & 67.3 & 72.2 & 3.9 \\
\hline Dhoke khabba & 56.4 & 58.2 & 60.1 & 52.8 & 56 & 62.2 & 65.1 & 67.8 & 58.7 & 64.6 & 67.8 & 52.8 & 60.2 & 4.7 \\
\hline Chaklala village nazir abad & 48.5 & 57.9 & 60.1 & 66.7 & 71.6 & 72.9 & 54.0 & 63.2 & 55.0 & 52.3 & 72.9 & 48.5 & 60.2 & 8.3 \\
\hline Residential area DHA-1 & 50.1 & 58.4 & 54.2 & 56.5 & 49.3 & 61.2 & 55.6 & 52.9 & 60.2 & 54.6 & 61.2 & 49.3 & 55.3 & 4.0 \\
\hline Hazarat abbas colony morghah & 61.5 & 51.2 & 52.4 & 50.2 & 60.9 & 66.3 & 68.8 & 56.7 & 54.4 & 57.3 & 68.8 & 50.2 & 58.0 & 6.3 \\
\hline Lal kurti residential area & 59.6 & 58.4 & 64.2 & 59.2 & 66.5 & 67.4 & 55.8 & 52.6 & 60.1 & 68.3 & 68.3 & 52.6 & 61.2 & 5.2 \\
\hline Markazi jamia mosque & 77.9 & 78.2 & 75.2 & 80.2 & 73.4 & 75.2 & 76.0 & 91.0 & 78.3 & 76.5 & 91.0 & 73.4 & 78.2 & 4.9 \\
\hline Askari-11 residential area & 53.1 & 52.2 & 49.5 & 55.9 & 57 & 59.2 & 68.1 & 71.8 & 61.3 & 65.4 & 71.8 & 49.5 & 59.4 & 7.3 \\
\hline PIA colony & 55.6 & 56.2 & 50.8 & 48.4 & 45.4 & 53.6 & 58.8 & 52.1 & 51.5 & 50.6 & 58.8 & 45.4 & 52.3 & 3.9 \\
\hline Cantt view colony & 54.3 & 50.7 & 50.9 & 43.1 & 42.9 & 53.1 & 59.2 & 53.2 & 54.1 & 51.2 & 59.2 & 42.9 & 51.3 & 5.0 \\
\hline Ilyaas colony misrial road & 50.0 & 56.5 & 53.7 & 48.2 & 49.6 & 54.2 & 58.6 & 53.5 & 51.8 & 52.4 & 58.6 & 48.2 & 52.9 & 3.2 \\
\hline Officers colony main entrance & 55.4 & 54.2 & 57.1 & 52.6 & 48.7 & 47.9 & 56.4 & 57.2 & 58.6 & 49.5 & 58.6 & 47.9 & 53.8 & 3.9 \\
\hline Railway scheme-7 & 66.9 & 65.5 & 70.4 & 66.4 & 67.3 & 80.7 & 71.9 & 64.5 & 63.1 & 63.8 & 80.7 & 63.1 & 68.1 & 5.2 \\
\hline Khayabane sir syyed area & 56.4 & 54.3 & 55.6 & 64.2 & 66.1 & 53.6 & 67.4 & 58.3 & 60.0 & 55.1 & 67.4 & 53.6 & 59.1 & 5.1 \\
\hline Bangash colony residential area & 64.5 & 70.1 & 68.1 & 65.8 & 64.1 & 63.9 & 66.2 & 68.3 & 64.3 & 63.7 & 70.1 & 63.7 & 65.9 & 2.2 \\
\hline Workshopi mohallah area & 58.2 & 56.7 & 62.2 & 55.2 & 49.8 & 50.3 & 56.9 & 60.1 & 61.4 & 61.0 & 62.2 & 49.8 & 57.2 & 4.4 \\
\hline Eid gah sihaam residential area & 43.5 & 45.6 & 53.2 & 52.1 & 48.2 & 38.8 & 55.2 & 50.1 & 42.4 & 47.5 & 55.2 & 38.8 & 47.7 & 5.2 \\
\hline
\end{tabular}

Table 3. Results from noise measurements on schools and colleges

\begin{tabular}{lllllllllllllll}
\hline \hline School/college & 1 & 2 & 3 & 4 & 5 & 6 & 7 & 8 & 9 & 10 & Max & Min & Mean & SD \\
\hline Govt.College for womens/town & 65.3 & 64.8 & 63.1 & 77.8 & 67.8 & 70.8 & 68.8 & 68.7 & 70.7 & 72.2 & 77.8 & 63.1 & 69.0 & 4.2 \\
Divisional public school & 71.3 & 73.6 & 69.6 & 68.9 & 70.6 & 72.3 & 68.8 & 69.2 & 70.2 & 72.1 & 73.6 & 68.8 & 70.7 & 1.6 \\
Asghar maal degree college & 70.9 & 68.2 & 64.6 & 66.3 & 70.0 & 68.5 & 65.1 & 62.3 & 61.5 & 60.0 & 70.9 & 60.0 & 65.7 & 3.7 \\
Army public school adiala road & 82.1 & 80.6 & 81.5 & 79.9 & 84.6 & 82.3 & 80.0 & 85.6 & 89.8 & 90.1 & 90.1 & 79.9 & 83.7 & 3.8 \\
Gordon college main gate & 80.8 & 81.0 & 82.4 & 79.8 & 84.6 & 90.1 & 90.2 & 88.9 & 90.1 & 82.2 & 90.2 & 79.8 & 85.0 & 4.3 \\
Gordon college inside & 79.5 & 80.1 & 77.8 & 79.8 & 79.6 & 78.6 & 77.5 & 76.8 & 78.3 & 80.2 & 80.2 & 76.8 & 78.8 & 1.2 \\
Army public school westridge-iii & 80.8 & 81.0 & 82.4 & 79.8 & 84.6 & 90.1 & 90.2 & 88.9 & 90.1 & 82.2 & 90.2 & 79.8 & 85.0 & 4.3 \\
F.G Boys school Peshawar road & 82.9 & 77.4 & 75.4 & 71.7 & 84.6 & 79.3 & 80.0 & 87.9 & 90.1 & 70.9 & 90.1 & 70.9 & 80.0 & 6.5 \\
Women college dhoke hassu & 81.9 & 76.1 & 94.4 & 81.7 & 78.8 & 77.8 & 81.0 & 92.2 & 73.3 & 88.1 & 94.4 & 73.3 & 82.5 & 6.9
\end{tabular}

Table 4. Results from noise measurements in hospitals

\begin{tabular}{lllllllllllllll}
\hline \hline Hospital & 1 & 2 & 3 & 4 & 5 & 6 & 7 & 8 & 9 & 10 & Max & Min & Mean SD \\
\hline Central hospital main gate & 82.0 & 73.3 & 79.1 & 68.5 & 77.6 & 68.4 & 70.4 & 72.5 & 84.2 & 73.2 & 84.2 & 68.4 & 74.9 & 5.5 \\
Central hospital inside & 65.2 & 64.5 & 66.3 & 58.4 & 60.0 & 58.1 & 67.6 & 69.2 & 55.4 & 54.2 & 69.2 & 54.2 & 61.9 & 5.3 \\
Holy family hospital inside & 63.2 & 63.4 & 62.1 & 61.6 & 64.9 & 76.8 & 71.4 & 67.5 & 63.8 & 65.0 & 76.8 & 61.6 & 66.0 & 4.8 \\
Holy family hospital main gate & 72.2 & 78.3 & 80.3 & 77.1 & 74.9 & 73.6 & 71.9 & 70.0 & 84.4 & 80.3 & 84.4 & 70.0 & 6.3 & 4.6 \\
Fauji foundation hospital & 54.3 & 50.7 & 50.9 & 45.1 & 48.9 & 53.1 & 59.2 & 55.2 & 52.1 & 56.2 & 59.2 & 45.1 & 52.6 & 4.0 \\
AFIC,CMH & 55.3 & 54.9 & 52.8 & 56.3 & 50.6 & 53.7 & 59.5 & 57.6 & 55.7 & 59.2 & 59.5 & 50.6 & 55.6 & 2.8 \\
Military hospital & 60.4 & 63.4 & 63.1 & 59.8 & 64.9 & 70.2 & 68.7 & 67.5 & 63.8 & 62.6 & 70.2 & 59.8 & 64.4 & 3.4 \\
\hline \hline
\end{tabular}


Table 5. Results from noise measurements at bus stands, airport and railway stations

\begin{tabular}{lcccccccccccccc}
\hline \hline Site & 1 & 2 & 3 & 4 & 5 & 6 & 7 & 8 & 9 & 10 & Max & Min & Mean & SD \\
\hline Pir Wadhai bus stand & 82.6 & 80.4 & 82.2 & 85.3 & 68.4 & 80.3 & 72.8 & 74.1 & 75.9 & 68.1 & 85.3 & 68.1 & 77.0 & 6.1 \\
Pir Wadhai bus stand & 80.2 & 78.7 & 78.6 & 79.9 & 78.3 & 80.2 & 81.2 & 78.2 & 80.6 & 86.4 & 86.4 & 78.2 & 80.2 & 2.4 \\
main entrance & & & & & & & & & & & & & \\
Islamabad & 59.2 & 67.4 & 72.6 & 70.7 & 71.9 & 74.0 & 69.1 & 77.2 & 101.2 & 102.5 & 102.5 & 59.2 & 76.6 & 14.1 \\
International airport & & & & & & & & & & & & & & \\
Chaklala railway station & 50.0 & 43.8 & 56.2 & 53.2 & 71.0 & 72.4 & 62.2 & 53.7 & 51.1 & 50.8 & 72.4 & 43.8 & 56.4 & 9.3 \\
Sowan wagon stand & 65.5 & 60.6 & 70.4 & 62.3 & 61.9 & 67.5 & 83.4 & 70.4 & 66.8 & 72.8 & 83.4 & 60.6 & 68.2 & 6.7 \\
Rawalpindi & 78.3 & 79.0 & 78.6 & 78.8 & 79.3 & 76.8 & 75.4 & 79.6 & 80.1 & 81.0 & 81.0 & 75.4 & 78.69 & 1.61 \\
Railway station outside & & & & & & & & & & & & & & \\
Rawalpindi & & & & & & & & & & & & & & \\
Railway station inside & 77.6 & 76.4 & 73.8 & 72.3 & 75.9 & 76.3 & 77.7 & 77.4 & 76.5 & 75.7 & 77.7 & 72.3 & 75.96 & 1.72 \\
Pir Wadhai bus stand & & & & & & & & & & & & & &
\end{tabular}

Table 6. Results from noise measurements in markets and shopping plazas

\begin{tabular}{lcccccccccccccc}
\hline \hline Market/plazas & 1 & 2 & 3 & 4 & 5 & 6 & 7 & 8 & 9 & 10 & Max & Min & Mean SD \\
\hline Food street cricket stadium & 67.7 & 59.4 & 71.5 & 67.1 & 68.9 & 72.9 & 70.0 & 66.0 & 69.8 & 68.5 & 72.9 & 59.4 & 68.2 & 3.7 \\
Shamas abad furniture market & 64.5 & 69.5 & 68.5 & 63.8 & 62.4 & 71.4 & 64.7 & 64.0 & 63.2 & 66.4 & 71.4 & 62.4 & 65.8 & 3.0 \\
Commercial market sat/town & 68.9 & 65.4 & 67.3 & 64.3 & 69.1 & 72.5 & 73.4 & 74.3 & 75.1 & 75.9 & 75.9 & 64.3 & 70.6 & 4.2 \\
Rahman abad choak market & 75.2 & 76.4 & 68.8 & 63.6 & 72.6 & 73.1 & 74.7 & 76.6 & 65.9 & 70.0 & 76.6 & 63.6 & 71.7 & 4.5 \\
National market satellite town & 66.5 & 67.3 & 70.1 & 72.5 & 60 & 62.6 & 71.7 & 70.3 & 66.8 & 78.3 & 78.3 & 60.0 & 68.6 & 5.2 \\
Chaklala market & 54.6 & 53.8 & 59.1 & 69.0 & 55.1 & 59.4 & 55.2 & 66.7 & 58.5 & 56.4 & 69.0 & 53.8 & 58.8 & 5.2 \\
Sadar kamran market & 75.4 & 76.2 & 75.8 & 79.6 & 77.9 & 80.2 & 81.0 & 79.8 & 80.3 & 78.5 & 81.0 & 75.4 & 78.5 & 2.1 \\
Bohar bazaar & 75.0 & 69.7 & 64.4 & 70.4 & 68.7 & 70.3 & 74.4 & 80.1 & 67.3 & 72.2 & 80.1 & 64.4 & 71.3 & 4.4 \\
Wesrtidge market & 78.2 & 78.4 & 77.6 & 80.1 & 79.6 & 78.8 & 76.9 & 76.5 & 81.2 & 80.1 & 81.2 & 76.5 & 78.7 & 1.5 \\
Westridge market post office & 76.4 & 76.8 & 81.0 & 79.2 & 78.6 & 78.4 & 79.3 & 74.8 & 76.7 & 77.9 & 81.0 & 74.8 & 77.9 & 1.8 \\
Khayabane sir syyed market & 80.8 & 80.2 & 70.8 & 67.3 & 71.1 & 69.8 & 72.6 & 73.4 & 71.3 & 73.7 & 80.8 & 67.3 & 73.1 & 4.3 \\
\hline \hline
\end{tabular}

Table 7. National environmental quality standards (NEQS) for noise effective from $1^{\text {st }}$ July, 2012

\begin{tabular}{lll}
\hline \hline Category of area/zone & \multicolumn{2}{c}{$\mathrm{dB}(\mathrm{A}) \mathrm{L}_{\mathrm{eq}}$} \\
\cline { 2 - 3 } & Day time & Night time \\
\hline Residential area & 55 & 45 \\
Commercial area & 65 & 55 \\
Industrial area & 75 & 65 \\
Silence zone & 50 & 45 \\
\hline \hline
\end{tabular}

The Gazette of Pakistan, extra, November 26, 2010 (Part-II), SRO 1064(1)/2010. can cause harmful effects on human health, animals and the environment. Motor vehicular traffic using pressure horns, musical systems and ill tuned engins are the main source of noise pollution at main roads as well as residential areas, main entrances of hospitals and educational institutions situated near roads. The problem of traffic noise created by these vehicles is a significant source of noise pollution in areas near roads and bus stands. Air conditioners, fans and generators cause noise pollution to some extent in markets and shopping plazas. 
Table 8. WHO guideline values for community noise in specific environments, April 1999

\begin{tabular}{lll}
\hline \hline Specific environment & $\begin{array}{l}\mathrm{L}_{\mathrm{eq}} \\
{[\mathrm{dBA}]}\end{array}$ & $\begin{array}{l}\mathrm{L}_{\max }, \text { fast } \\
{[\mathrm{dBA}]}\end{array}$ \\
\hline Outdoor living area & 55 & - \\
Dwelling, indoors & 35 & 45 \\
Inside bedrooms & 30 & 45 \\
Outside bedrooms & 45 & 60 \\
School class rooms and & 35 & - \\
Pre-schools, indoors & & \\
& 30 & 45 \\
Pre-school bedrooms, indoors & 55 & - \\
School, playground outdoor & 30 & 40 \\
Hospital, ward rooms, indoors & 70 & 110 \\
Industrial, commercial shopping & & \\
and traffic areas, indoors \& outdoors & & \\
Ceremonies, festivals and & 100 & 110 \\
entertainment events & & \\
Public addresses, indoors and outdoors & 85 & 110 \\
Music through headphones/ earphones & 85 & 110 \\
\hline \hline
\end{tabular}

During load shedding of electricity, mostly generators are used to generate electricity. These are creating too much noise in commercial areas. Excessive use of loudspeakers and musical equipments in musical centers is causing noise pollution in markets and shopping plazas. Overcrowding of people is creating noise in markets, schools, colleges, hospitals, bus stands airports and railway stations. Unplanned urbanisation has severly damaged the natural green areas of the city. These all are contributing to noise pollution, which can cause health problems to the exposed population of the city. Present study will certainly divert attention of the concerned implementing authorities to understand the severity of the noise pollution problem of the city and take proper remedial measures.

\section{Recommendations}

- To wear ear protection, while working in noisy conditions is an effective way to manage noise. Vehicles and factory machines need to be maintained properly and checked from time to time.

- Sound insulation at the top of the roof and addition of a layer of plasterboard or wood to the dividing wall can provide a protection from the noise.

- A comprehensive campaign should be launched to create public awareness about noise pollution.

- Effective solution for noise pollution is to plant bushes and trees around the sound generating sources. Planned housing schemes should be allowed which must have green areas.

- Use of loudspeakers at public places, use of pressure horns and sound systems in buses and other transport vehicles should be banned and strict laws should be imposed against them.

- For overcoming the effects of noise pollution latest active noise control (ANC) technologies should be used such as white noise machine. This device converts the unbearable noise into pleasant sound.

\section{References}

Alam, M.J.B., Rauf, A.F.M.A., Ahmed, M.F. 2001. Traffic induced noise pollution in Dhaka City. Journal of Civil Engineering, CE29: 55-63.

Aslam, M.J., Aslam, M.A., Batool, A. 2008. Effect of noise pollution on hearing of public transport drivers in Lahore city. Pakistan Journal of Medical Sciences, 24: 142-146.

Clark, C., Stephen, A.S. 2005. The effect of aircraft and road traffic noise on children's reading. Literacy Today, 44: 24-25.

Evans, G.W. et al. 2001. Community noise exposure and stress in children. Journal of the Acoustical Society of America, 109: 1023-1027.

Havas, V. 2006. Noise, the invisible pollution. Current Health, 32: 10-11.

Khan, M.W., Memon, M.A., Khan, M.N., Khan, M.M. 2010. Traffic noise pollution in Karachi, Pakistan. Journal of Liaquat University of Medical and Health Sciences, 9: 114-120.

Mia, M.Y., Hossain, Md.U., Farzana, S. 2012. Measurement of noise intensity in Tangail municipal area, Bangladesh. Pakistan Journal of Scientific and Industrial Research, Ser. A: phys. sci., 55: 92-97.

Murthy, V.K., Majumder, A.K., Khanal, S.N., Subedi, D.P. 2007. Assessment of traffic noise pollution at 
banepa, a semi urban town of Nepal. Kathmandu University Journal of Science, Engineering and Technology, 1: 1-9.

NEQS, 2010. National environmental quality standards for noise, The Gazette of Pakistan, Extra., (SRO 1064(I) / 2010), for noise, (Part-II) $26^{\text {th }}$ November, 2010.

Staples, S.L. 1997. Public policy and environmental noise, Modeling exposure or understanding effects. American Journal of Public Health, 87: 2063-2067.

WHO, 2001. Fact Sheet No. 258. Geneva, WHO Press. http://www.who.int/mediacentre/factsheets/ fs $258 /$ en/.

WHO, 1999. Organization Guidelines for Community Noise, April 1999, London, UK.

WWW.World Gazetteer.com, Top 100 most populous cities in Asia 2010. 